\title{
Topical nicotinic receptor activation improves wound bacterial infection outcomes and TLR2-mediated inflammation in diabetic mouse wounds
}

\author{
Mari Kishibe, MD, PhD ${ }^{1,2}$, Tina M. Griffin, MLIS ${ }^{1,2}$, Melissa Goslawski, MD ${ }^{5}$, James \\ Sinacore, $\mathbf{P h D}^{4,5}$, Sascha A. Kristian, $\mathbf{P h D}^{1,2}$, and Katherine A. Radek, $\mathbf{P h D}^{1,2,3,5}$ \\ ${ }^{1}$ The Burn and Shock Trauma Research Institute, Loyola University Chicago, Health Science \\ Division, Maywood, Illinois, USA \\ 2Department of Surgery, Loyola University Chicago, Health Science Division, Maywood, Illinois, \\ USA \\ ${ }^{3}$ Infectious Disease and Immunology Research Institute in the Department of Microbiology and \\ Immunology, Loyola University Chicago, Health Science Division, Maywood, Illinois, USA \\ ${ }^{4}$ Department of Public Health Science, Loyola University Chicago, Health Science Division, \\ Maywood, Illinois, USA \\ ${ }^{5}$ Stritch School of Medicine, Loyola University Chicago, Health Science Division, Maywood, \\ Illinois, USA
}

\begin{abstract}
The cholinergic anti-inflammatory pathway can directly affect skin antibacterial responses via nicotinic acetylcholine receptors (nAChRs). In particular, a $7 \mathrm{nAChR}$ (CHRNA7) present in the epidermis modulates the host response to wounding and/or wound bacterial infection. While physiologic inflammation is required to initiate normal wound repair and can be triggered by Tolllike receptor (TLR) activation, chronic inflammation is frequently observed in diabetic wounds, and can occur, in part, via excessive TLR2 activation or production. Consequently, this can delay physiologic wound healing responses and increase diabetic host susceptibility to bacterial infection. In this study, we demonstrate that topical $\mathrm{nAChR}$ activation diminishes bacterial survival and systemic dissemination in a mouse model of diabetic wound infection, while reducing wound TLR2 production, relative to control mice. We further determined that the AMP activity of diabetic mouse wounds is increased compared to control mice, but this effect is lost following topical nAChR activation. Finally, we observed that human diabetic wounds exhibit impaired a 7
\end{abstract}

Correspondence and Reprints: Katherine A. Radek PhD, Loyola University Chicago, Health Sciences Division, The Burn and Shock Trauma Research Institute, Department of Surgery, Center for Translational Research and Education, Room 326, 2160 S. First Avenue, Maywood, IL 60153, Phone: (708)327-2360; FAX: (708)327-2813, kradek1 @luc.edu. AUTHOR CONTRIBUTIONS

M.K. performed most mouse experiments, analyzed results and contributed to manuscript writing. T.M.G. performed mouse experiments and several subsequent analyses. M.G. performed the human chronic wound analyses. J.S. was involved in the biostatistical analyses. S.A.K. was involved in the generation of RDA data and data analyses. K.A.R directed the study, performed experiments, and wrote the final version of the manuscript.

COMPETING FINANCIAL INTERESTS

The authors declare no competing financial interests. 
nAChR (CHRNA7) abundance and localization relative to human control (non-diabetic) skin. These findings suggest that topical administration of $\mathrm{nAChR}$ agonists may improve wound healing and infection outcomes in diabetic wounds by dampening TLR2-mediated inflammation and AMP response, and that the diabetic microenvironment may promote aberrant CHRNA7 production/ activation that likely contributes to diabetic wound pathogenesis.

\section{Keywords}

diabetes; skin wounds; infection; antimicrobial peptides; nicotinic receptors; Toll-like receptor 2

\section{INTRODUCTION}

Diabetic wounds diminish the quality of life for millions of patients, and pose a significant economic burden on society that exceeds \$10B annually in the US alone. Nearly $15-20 \%$ of all patients with diabetes develop lower extremity wounds, and diabetes accounts for $>60 \%$ of all lower extremity amputations in the US (1-3). Furthermore, bacterial infection is considered a critical component for impaired diabetic wound healing (4), due to compromised leukocyte function, accompanying vascular diseases, inadequate glucose control and altered host antimicrobial responses (1). Consequently, research exploring the mechanisms by which stress responses, specifically cholinergic receptors, influence wound healing and infection within the diabetic population is of critical importance from a financial and psychosocial perspective.

In the past several years, research in the areas of neuroendocrine responses related to skin function and infection has expanded considerably. The neuroendocrine system is known to modulate systemic and local immune responses, in part, through the cholinergic pathway, which spans several environments to control inflammation via nicotinic acetylcholine receptors (nAChRs) during stress (i.e., infection, tissue damage, disease states). With regard to inflammation, the neuronal cholinergic anti-inflammatory pathway was originally found to control inflammation by the a $7 \mathrm{nAChR}$ (CHRNA7) on cytokine producing cells during infection and tissue damage through the release of acetylcholine ( $\mathrm{ACh}$ ) from neuronal tissues (5). Studies from our laboratory and others using animal and human models have determined that stress and activation of epidermal nAChRs directly influence tissue repair processes and susceptibility to infection through modulation of skin antimicrobial responses (6-8). A more recent study also determined that CHRNA7 activation can promote diabetic wound healing by suppressing TNF-a production by advanced glycation end products, which may be linked with the blockage of NF- $\kappa \mathrm{B}$ activation in macrophages (9), further implicating a role for $\mathrm{nAChR}$ activation in diabetic wound healing pathology.

Impaired antimicrobial peptide (AMP) activity is one mechanism behind susceptibility to microbial infections and pathologic inflammation, as AMPs stimulate inflammation, provide direct microbicidal activity, and maintain skin barrier integrity (10). AMP dysregulation can promote the pathogenesis of inflammatory skin diseases and compromises the normal wound healing process in humans (11-13). Toll-like Receptor 2 (TLR2) is activated after skin wounding or Staphylococcus aureus infection, and plays an important role in the 
induction of AMP production and bacterial clearance, as well as stimulating wound closure $(14,15)$. TLR2 recognizes components of Gram-positive bacteria by facilitating heterodimers with TLR1 or TLR6 $(16,17)$, leading to the recruitment of the intracellular proteins necessary for induction of pro-inflammatory cytokine and AMP production (14, 15). Importantly, TLR 2 expression, signaling, and functional activation are increased in type 2 diabetes human subjects (18) and experimental skin wounds in diabetic $d b / d b$ mice (19). This positive feedback loop leads to excess wound inflammation and delayed wound closure. Furthermore, the cholinergic anti-inflammatory pathway was recently implicated in obesity-induced inflammation and insulin resistance (20), characteristic of diabetic wound patients.

We previously published that keratinocyte nAChR activation dampens TLR2-mediated migration and pro-inflammatory cytokine and AMP production using the TLR2/6 ligand, MALP-2 (macrophage activating lipoprotein-2), which can be restored by a CHRNA7selective antagonist. We further determined that this response occurred through the NF- $\kappa B$ and Erk1/2 pathways during early and late wound healing. In a mouse model of $S$. aureus wound infection, topical nAChR activation reduced wound AMP and TLR2 production to augment bacterial survival in wild-type mice, as AMP induction and TLR2 production are known to facilitate bacterial clearance during skin infection (21). Therefore, we hypothesized that nAChR activation would reduce TLR 2 responses in a model of diabetic wound infection to promote healing and reduce bacterial survival, as TLR2 abundance and excess inflammation are believed to impede normal wound healing responses and increase bacterial susceptibility in this model of impaired wound healing. Our findings emphasize the potential benefit of epidermal nAChR activation on aberrant epidermal TLR2-mediated responses to improve diabetic wound healing and infection outcomes, and identify a potential defect in epidermal cholinergic responses in diabetic patients.

\section{METHODS}

\section{Murine MRSA wound infection model.}

Mice homozygous for the diabetes spontaneous mutation $d b(\operatorname{Lepr} d b / d b)$ and heterozygous control mice ( Lepr $\left.^{d b /+}\right)$ (8-10-week-old males) were purchased from Jackson Laboratories. All animal experiments were performed with the approval of the Loyola University Chicago, Health Sciences Campus IACUC. Three days prior to treatment start, dorsal mouse fur was plucked under isofluorane anesthesia and immediately depilated. Vehicle $(0.1 \%$ ethanol in cell-culture tested water) or $10^{-9} \mathrm{M}$ nicotine (Sigma) were topically applied to dorsal skin twice a day for 3 days. Subsequently, skin wounds were induced with 3-mm biopsy punches at 6 sites, and then $10 \mu \mathrm{l}$ of either vehicle (sterile saline) or the methicillin-resistant $S$. aureus (MRSA) USA300 strain ( $10^{6} \mathrm{CFU}$ total) was applied topically to each wound. On day 1 post-wounding, the mice were humanely euthanized and their wounds and kidneys were excised and prepared for subsequent analyses. To quantify the bacterial burden, one wound and kidney per mouse was homogenized in sterile PBS with $1 \mathrm{~mm}$ zirconia beads in a mini bead-beater (BioSpec Products). The homogenates were serially diluted in PBS and plated on Todd-Hewitt agar plates. After overnight incubation of the agar plates, the bacterial colonies were counted to quantify the colony forming units $\mathrm{CFU} / \mathrm{ml}$ homogenate. The rest of 
the skin biopsies was immediately frozen after excision on dry ice and stored at $-80^{\circ} \mathrm{C}$ until use.

\section{Enzyme-linked immunosorbent assay.}

The levels of IL-6, MIP-2, IL-10, and IL-17A in skin wound homogenates were determined using ELISA kits (PepProTech) according to the manufacturer's protocols.

\section{Western blot.}

Wounds were pulverized using a liquid nitrogen cooled biopulverizer (Biospec). Powdered tissues was then dissolved in in RIPA buffer containing 10 units $/ \mathrm{mL}$ collagenase (SigmaAldrich) and HALT phosphatase and protease inhibitor cocktail (Pierce Biotechnology) and incubated for $1 \mathrm{hr}$ at $37^{\circ} \mathrm{C}$. Afterwards, the homogenates were sonicated on ice for $10 \mathrm{sec}$ and then spun at $17,000 \times g$ for 5 minutes. The supernatants were transferred to new tubes and stored at $-80^{\circ} \mathrm{C}$ until use. Protease inhibitor was present in all reagents. The protein samples were heated at $90^{\circ} \mathrm{C}$ for 5 min prior to separation in a 4-20\% gradient minigel (Bio Rad). Proteins in gels were transferred to PVDF membranes and immunoblotted with the following antibodies at 1:1000 or 1:2000 dilution: Rabbit polyclonal anti-human $\beta 2$ microglobulin (Pierce Biotechnology), mouse monoclonal anti-human $\beta$-actin (Santa Cruz Biotechnology) for cytosolic extracts, mouse monoclonal anti-human TATA box (Millipore) for nuclear extracts, rabbit polyclonal anti-TLR2 (Abcam), mouse monoclonal anti-CD14 (Santa Cruz Biotechnology), rabbit polyclonal anti-MyD88 (Cell Signaling), anti-mouse CHRNA7 (Abcam), and mouse monoclonal anti- $\beta$-actin at 1:1000 for culture cell or mouse skin lysates. Goat anti-rabbit HRP or goat anti-mouse HRP (Vector Laboratories) at 1:5000 were used as secondary antibodies. Visualization of the immunoreactive bands was performed by enhanced chemiluminescence (Pierce Biotechnology). Densitometry of bands was analyzed using Image $\mathbf{J}$ software (22).

\section{HPLC Purification of Skin Extracts.}

Skin samples were placed in $1 \mathrm{~N}$ acetic acid for at least 24 hours at $4^{\circ} \mathrm{C}$, homogenized, lyophilized and then dissolved in water. High Pressure Liquid Chromatography (HPLC) peptide separation was then performed using a C18 column ( $8 \mu \mathrm{M}$, ST 4.6/250; Thermo Scientific). Equilibration was done in $0.1 \%$ trifluoroacetic acid (TFA). Peptides were eluted with an acetonitrile gradient (10-100\% over $40 \mathrm{~min}$ at $2 \mathrm{ml} / \mathrm{min}$, fractions collected from 10-30 minutes) (23). All fractions ( $2 \mathrm{ml}$ each) were lyophilized and reconstituted in $50 \mu \mathrm{l}$ of sterile water. The samples were then re-lyophilized and resuspended in $20 \mu \mathrm{l}$ sterile water. Samples were then normalized for peptide content.

\section{Antimicrobial Radial Diffusion Assay.}

Recombined HPLC fractions were evaluated using a radial diffusion assay (RDA), as we described $(6,21)$. Antimicrobial activity was assessed against $S$. aureus $(S$. aureus $\Delta m p r F)$. Bacteria were grown overnight in Tryptic Soy Broth (TSB) at $37^{\circ} \mathrm{C}$. The overnight cultures were then diluted 1:100, grown to mid-exponential phase, and spectrophotometrically adjusted to the desired CFU concentration in TSB. Agar plates for the RDAs were prepared as previously described (24). Briefly, $0.5 \%$ tryptone medium with $1 \%$ SeaKem GTG 
Agarose (Cambrex Corporation) was autoclaved. The agar was cooled to $45^{\circ} \mathrm{C}$ and the bacteria added to an endconcentration of $\sim 4 \times 10^{5} \mathrm{CFU} / \mathrm{ml}$. Immediately thereafter, $10 \mathrm{~mL}$ of the liquid agar with the bacteria was poured into square agar plates. After the agar solidified, the plates were refrigerated to inhibit bacterial growth. Then, 36 holes with defined size were placed into the agar plates by using sterile $1000-\mu \mathrm{L}$ pipette tips and vacuum suction. Finally, $2 \mu \mathrm{L}$ of the skin peptide samples were placed into the holes and the plates incubated for $24 \mathrm{~h}$ at $37^{\circ} \mathrm{C} .2 \mu \mathrm{L}$ sterile water and synthetic catestatin AMP (100 $\mu \mathrm{M}$, GeneScript) were used as the negative and positive controls, respectively. The zone of inhibition of bacterial growth (area) was quantified using ImageJ Software (22).

\section{Laser capture microdissection (LCM) and quantitative PCR.}

Frozen sections mounted onto membrane-slides (Carl Zeiss Microscopy) were fixed in 70\% ethanol, and washed with RNase-free distilled water for $5 \mathrm{~min}$. Sections were stained with Mayer's hematoxyline, and then rinsed with RNase-free water. After that, dehydration was performed in a quick increasing ethanol series. LCM was performed using a PALM Micro Beam System (Zeiss Instruments). Samples from each group were collected and pooled per group into an Adhesive Cap 500 opaque (Carl Zeiss Microscopy) and stored in QIAzol Lysis Reagent (QIAGEN) at $-80^{\circ} \mathrm{C}$. RNA extraction was performed using the miRNeasy Micro Kit (QIAGEN). Complementary DNA was synthesized using iScript cDNA Synthesis Kit (Bio Rad). Quantitative real time RT-PCR was performed in triplicate using TaqMan Universal PCR Master Mix (Life Technologies) on a StepOnePlus Real-Time PCR System (Life Technologies). Fluorogenic probes and primers were used to detect: IL-6 (Mm00446190_m1), MIP-2 (Mm00436450_m1), and TLR2 (Mm00442346_m1) for mouse samples (Life Technologies). Expression of target gene was normalized to $\beta$-actin. Expression was analyzed by the $2^{-\Delta \Delta \mathrm{Ct}}$ method.

\section{Immunofluorescence with human diabetic wounds.}

Discarded tissue was obtained from patients following amputation due to diabetic, nonhealing wounds with diabetic wounds $(\mathrm{N}=5$; male; white/Hispanic). Amputation procedures include amputation of digits of the lower extremity, transmetatarsal amputation of the foot, below knee amputation, through knee amputation or above knee amputation. Control skin was collected from tissue discarded following elective surgical procedures $(\mathrm{N}=5)$. Tissue was collected under the IRB protocol 202919 approved by the Loyola Institutional Review Board. A $12 \mathrm{~mm}^{2}$ area of skin was obtained from an area of active infection without evidence of tissue necrosis. Skin was embedded in OCT. 8- $\mu \mathrm{m}$ sections were prepared and subjected to immunofluorescence. To that aim, the sections were washed with wash buffer (Dako), fixed with 4\% paraformaldehyde in PBS, and blocked with protein block solution (3\% albumin in Tris buffered saline with Tween (TBST)). Slides were incubated with mouse monoclonal anti-TLR2 antibody (Chemicon) diluted at 1:150 or Rabbit anti-CHRNA7 $(\mathrm{R} \& \mathrm{D})$ diluted at $1: 200$ overnight at $4^{\circ} \mathrm{C}$. Mouse IgG (Invitrogen) or rabbit $\operatorname{IgG}$ (Abcam) were used as negative controls. Donkey anti-mouse FITC (Millipore) or donkey anti-rabbit antibody Cy3 (Jackson Immuno Research Laboratories) or were used as secondary antibodies. Nuclei were stained using Prolong Antifade Gold with DAPI (Life Technologies). Images were captured with an EVOS FL Digital Microscope (Electron Microscopy Sciences). 


\section{Statistical analyses.}

Values were expressed as means $\pm \mathrm{sem}$. The unpaired t-test or unpaired Mann-Whitney test were applied to analyze the differences between two groups. ANOVA with appropriate Sidak's or Bonferroni's post-test were used for multiple comparisons. Analyses were performed using GraphPad Prism for Windows. $P$ values smaller than 0.05 were considered to be statistically significant. All statistical analyses were confirmed with Dr. Jim Sinacore, Ph.D., a biostatistician at Loyola University Chicago.

\section{RESULTS}

\section{Topical nicotine reduces bacterial survival and dissemination in diabetic MRSA infected mouse wounds}

Bacterial infection, which may eventually systemically spread, is considered a critical component for impaired diabetic wound healing, in part, due to impaired host antimicrobial responses $(1,25,26)$. Importantly, TLR2 expression, signaling, and functional activation are increased in type 2 diabetes human subjects (27) and diabetic ( $d b / d b)$ mouse wounds (19). Robust bacterial colonization promotes persistent activation of TLR2 signaling, which can lead to pathologic inflammation $(13,14,19,28)$. As nAChRs are known to diminish inflammation, we hypothesized that pharmacologic nAChR activation may help reduce the bacterial burden in a diabetic wound model of $S$. aureus infection. To test this, we subjected control $\left(\right.$ Lepr $\left.^{+/ d b}\right)$ or diabetic $\left(L e p r^{d b / d b}\right)$ mice to topical nicotine or vehicle treatment prior to excisional skin wounding and topical MRSA infection, as we previously described (21). Nicotine treatment increased wound size in day 1 uninfected wounds in diabetic Lepr $d b / d b$ mice relative to vehicle treatment (Fig. 1a), but was able to significantly reduce wound size at day 7 post-wounding (Fig. 1b). No change in wound size was observed in infected mouse wounds with nicotine treatment in diabetic mice, but it was detrimental for wound healing in control mice (Fig. 1b). In terms of bacterial survival, control mice treated with nicotine had a significantly increased bacterial burden in wounds $24 \mathrm{~h}$ after MRSA infection (Fig. 1c). In contrast, nicotine significantly reduced $S$. aureus MRSA survival in skin wounds (Fig. 1c) and dissemination into the kidney of $L e p r^{d b / d b}$ mice (Fig. 1d).

\section{Topical nicotine normalizes wound AMP activity and reduces pro-inflammatory IL-6 production in MRSA infected diabetic wounds.}

Local abundance, as well as peptide charge and hydrophobicity, are important factors that determine effectiveness of a particular AMP, and dictate an AMP's ability to efficiently kill microbes and stimulate later immune responses $(23,29)$. To first assess AMP activity and hydrophobic characteristics of wound extracts from control or diabetic wounds subjected to vehicle or nicotine treatment, HPLC was used to fraction wound homogenates prior to analysis of antimicrobial action. Peptides eluted at $10-55 \%$ acetonitrile, where AMPs exhibiting greater hydrophobicity eluted from the column at one-minute intervals with increasing percentages of acetonitrile. Fractions from each specimen were subjected to a radial diffusion assay (RDA) to assess their capacity to inhibit the growth of an AMPsusceptible mutant, $S$. aureus $\Delta m p r F$. In day 1 uninfected mouse wounds from control or $L e p r^{d b / d b}$ mice, we did not observe any statistical differences in AMP activity (Fig. 2a, c) of wound fractions between uninfected vehicle or nicotine treated mice, as measured by the 
diameter of bacterial growth inhibition (Fig. 2). However, we did observe a significant increase in AMP activity in fractions 7 and 14 of MRSA-infected $L e p r^{d b / d b}$ mice, as well as an increase in peptide hydrophobicity, relative to controls (Fig. 3a,b). In contrast, topical nicotine treatment normalized AMP activity and hydrophobicity to that of control mice (Fig. $3 \mathrm{c}, \mathrm{d})$.

To next determine if topical nAChR activation would alter AMP-dependent cytokine production, we quantified wound IL-6, IL-17A, TNF-a, and IL-10. We observed a significant reduction in IL-6 in infected diabetic mouse wounds after nicotine treatment, but no change in control wounds (Fig. 4a). No change was observed in IL-17A, a potent T-cell derived cytokine important for keratinocyte AMP induction, or TNF-a, a pro-inflammatory cytokine negatively regulated by AMPs (Fig. 4b,c). Although we observed a slight increase in IL-10 in control mouse wounds following nAChR activation, this effect was absent in diabetic mouse wounds (Fig. 4d). Thus, epidermal nAChR activation appears to dampen aberrant AMP activity and pro-inflammatory IL-6 in MRSA-infected $L$ epr ${ }^{d b / d b}$ mice exhibiting known pathologic inflammation and impaired wound healing responses.

\section{Topical nicotine reduces TLR2 and CHRNA7 protein production in MRSA infected diabetic wounds.}

We further investigated whether epidermal nAChR activation may be influencing wound antimicrobial and inflammatory responses through changes in TLR2 production in the context of wound infection. Membrane TLR2 $(100 \mathrm{kD})$ protein levels were markedly reduced by $\sim 50 \%$ in MRSA infected wounds in both control and $L e p r d b / d b$ mice (Fig. 5a,b). Furthermore, we detected 2 isoforms of the soluble TLR2 receptor $(54 \mathrm{kD}$ and $46 \mathrm{kD})$ in control mice (Fig. 5a,b). Soluble TLR2 consists mostly of the TLR2 extracellular domain, has been detected in plasma, breast milk and saliva $(30,31)$, and serves as a decoy receptor to prevent overactivation of membrane-bound $\operatorname{TLR} 2(28,32)$. Only the TLR2 membrane and $54 \mathrm{kD}$ soluble form were reduced in infected mouse wounds by nicotine treatment in control mice (Fig. 5a,b). Intriguingly, Lepr ${ }^{d b / d b}$ infected mouse wounds were deficient in the $54 \mathrm{kD}$ soluble TLR2, but we did observe a significant reduction in membrane TLR2 by topical nicotine (Fig. 5a,b). No statistical difference in the protein levels of the $46 \mathrm{kD}$ soluble TLR2 was observed in control or Lepr ${ }^{d b / d b}$ infected mouse wounds (Fig. 5a,b). Epidermal TLR2 production decreased with topical nicotine in infected wounds from both control and $L e p r^{d b / d b}$ mice. We further observed that epidermal CHRNA7 production increased with topical nicotine in infected control wounds, but decreased in infected diabetic wounds (Fig. $5 \mathrm{a}, \mathrm{c})$. These results suggest that TLR2 and TLR2-mediated wound healing responses are targeted by epidermal nAChR activation, and that the diabetic microenvironment changes the skin's responsiveness to cholinergic stimuli, and remains to be explored.

To next confirm whether the changes in TLR2 and IL- 6 were primarily in the epidermis, laser-capture microdissection was used to isolate RNA from epidermal keratinocytes in mouse wounds (Supplementary Fig. 1). Due to the low RNA yield isolated from the epidermis, it was necessary to pool the samples for each group. We determined that TLR2 and IL-6 epidermal gene expression appeared to be more abundant in uninfected diabetic mouse wounds compared to controls, and that topical nAChR activation appeared to reduce 
epidermal TLR2 and IL-6 more robustly in diabetic wounds than controls, similarly to our protein data in Figure 5. Future studies are necessary to robustly increase the number of mice in order to perform statistical analyses, but the data suggest that similar changes in TLR2 and IL-6 occur at both the RNA and protein level, and that these changes likely occur in the epidermis. Of note, we were unable to isolate epidermis from infected diabetic wounds, as these wound sections were not able to be captured by the laser. It was speculated that perhaps epidermal lipid content or another biochemical defect was more abundant in these wounds, rendering them too heavy for the laser capture. However, this concept needs to be further explored in the context of infected diabetic mouse wounds.

\section{CHRNA7 and TLR2 co-localization is altered in human diabetic wounds relative to control skin.}

Finally, we sought to establish proof-of-concept that human chronic wounds exhibited defects in epidermal nAChRs relative to TLR2. As compared to control skin, which exhibited positive staining of CHRNA7 primarily in the stratum corneum, skin from chronic wounds exhibited a more diffuse pattern of CHRNA7 (red) throughout the epidermis (Supplementary Fig. 2). TLR2 localization (green) was also more prominent in the dermis in control skin, as compared to chronic wounds, which expressed TLR2 in the more differentiated epidermal layers (Supplementary Fig. 2). These results demonstrate a defect in the localization of both CHRNA7 and TLR2 in the epidermis of chronic wounds, which likely influences TLR2-mediated responses involved in diabetic wound pathogenesis.

\section{DISCUSSION}

Our results can be simplified in our model of the TLR2/cholinergic axis in the context of cutaneous wound healing (Supplementary Fig. 3). TLR2 overactivation, as observed in diabetic subjects and mice, can promote hyper-inflammation and aberrant AMP production, resulting in impaired wound healing $(19,27,33)$. Our previous studies identified a novel mechanism for AMP regulation via the $\mathrm{nAChR}$ activation in response to skin infection (6). Here, we hypothesized that altered states of $\mathrm{nAChR}$ activation modulate TLR2 activation, upstream of AMP regulation, to diminish TLR2-dependent wound repair responses. We further hypothesized that this may be beneficial in models of chronic wounds, specifically diabetic wounds, where undesirable TLR2 activation and hyper-inflammation serves to impede wound closure and bacterial resistance $(19,27,34)$. Thus, selective nAChR agonists may have a significant therapeutic potential to improve wound healing in diabetic or other subsets of chronic wounds where infection and/or inflammation is in excess, consequently impeding normal wound healing processes.

Our results confirmed these hypotheses while also revealing that the presence of a TLR2 stimulus (i.e., infection) alters the wound response to nAChR activation. In MRSA infected wounds in control mice, nAChR activation dampens TLR2-mediated pro-inflammatory cytokine and AMP production, which likely contributes to greater bacterial survival and systemic dissemination into the kidney observed in nicotine treated mice (21). In contrast, topical nicotine reduces bacterial susceptibility in an infected diabetic skin wound along with the reduction of TLR2 production and the presence of alternative isoforms, suggesting 
that $\mathrm{nAChR}$ activation can improve wound healing outcomes in the presence of excess inflammation. We also observed that topical $\mathrm{nAChR}$ activation increased wound size in uninfected diabetic mouse wounds at Day 1 and infected control wounds at Day 7 postwounding. However, wound size was reduced only in uninfected diabetic mouse wounds at Day 7 post-wounding, indicating a selective nicotine effect in the presence or absence of infection. Alternatively, wound contractability may also account for the differences in lesion size between the mouse strains, which remains to be further explored in subsequent studies.

Excessive inflammation and frequent bacterial colonization are major contributing factors for intractable diabetic foot ulcers (25). Enhanced TLR2 expression and downstream activation pathways promotes a sustained inflammatory responses and dysfunctional wound healing in the diabetic human and mouse $(19,27,33)$. Consistent with another report (19), TLR2 was increased in migrating and proliferating epidermis in $L e p r d b / d b$ mouse wounds compared to controls. Topical nicotine markedly reduced TLR2 production in $L e p r^{d b / d b}$ mouse wounds, suggesting that $\mathrm{nAChR}$ activation may diminish TLR2-mediated responses to improve wound healing responses in diabetic wounds, including the observed normalization of wound AMPs in infected nicotine-treated diabetic mouse wounds. Along these lines, cathelicidin production is reduced in diabetic foot ulcers compared to healthy skin (35), which likely contributes to greater bacterial susceptibility and delayed wound reepithelialization. Interestingly, we observed a robust increase in epidermal cathelicidin expression in nicotine-treated $L e p r^{d b / d b}$ mice compared to vehicle treated or control animals (data not shown). This suggests that something specific about the diabetic phenotype or microenvironment permits a greater responsiveness to topical $\mathrm{nAChR}$ activation in terms of cathelicidin regulation, a mechanism that remains to be investigated.

While nicotine increased IL-10 in infected control wounds, this effect was lost in Lepr $r^{d b / d b}$ mice wounds. The nicotine metabolite, cotinine, was previously found to promote an IL-10dominated anti-inflammatory milieu (36). IL-17A, which induces AMP expression in keratinocytes (37), is increased by high glucose treatment in lymphocytes (38). Consistent with this, we observed greater IL-17A in $L e p r^{d b / d b}$ mice wounds compared to wild-type wounds ( 230\%). Since diabetic wounds exhibit reduced cathelicidin (35), increased IL-17A may serve as a compensatory mechanism to increase cathelicidin. Selective nAChR agonists may serve to "normalize" specific cytokines in diabetic wounds to improve the inflammatory microenvironment.

Although topical nicotine application was previously found to accelerate diabetic wound healing by augmenting angiogenesis using nanomolar concentrations (39), we observed a new mechanism by which nAChR activation dampens TLR2-mediated wound healing responses and influences bacterial survival/dissemination. Our study reveals not only the suppressive effect of nAChR activation on TLR2-mediated wound responses, but also new insights and potential drug targets to improve wound healing outcomes in diabetic and other subsets of chronic wounds. The observed increase in TLR2/ CHRNA7 co-localization in the epidermis also suggests that their proximity may explain potential paracrine effects by CHRNA7 activation on TLR2 responses, and that the altered distribution of CHRNA7 may be directly contributing to impaired wound healing and antimicrobial responses in diabetic patients, which remains to be further explored. 


\section{Supplementary Material}

Refer to Web version on PubMed Central for supplementary material.

\section{ACKNOWLEDGEMENTS}

Research reported in this publication was partially supported by the National Institute of Arthritis and Musculoskeletal and Skin Diseases of the National Institutes of Health under Award Number 1R01 ARO61497-01A1. This research was also supported, in part, by resources provided by the Northwestern University Skin Disease Research Center (NU-SDRC, Chicago, IL) through the NIH NIAMS P30-AR-057216 Award. Any opinions, findings, conclusions, or recommendations expressed in this material are those of the author(s) and do not necessarily reflect the views of the NU-SDRC or the NIH/NIAMS. This work was also supported by a 3M Wound Healing Society Foundation Fellowship Award to K.A.R. We would like to thank Dr. Richard C. Hershberger for collection of human tissue samples.

\section{REFERENCES}

1. Sen CK, Gordillo GM, Roy S, Kirsner R, Lambert L, Hunt TK, et al. Human skin wounds: a major and snowballing threat to public health and the economy. Wound repair and regeneration : official publication of the Wound Healing Society [and] the European Tissue Repair Society 2009;17(6): 763-71.

2. Brem H, Tomic-Canic M. Cellular and molecular basis of wound healing in diabetes. J Clin Invest 2007;117(5):1219-22. [PubMed: 17476353]

3. Reiber GE, Vileikyte L, Boyko EJ, del Aguila M, Smith DG, Lavery LA, et al. Causal pathways for incident lower-extremity ulcers in patients with diabetes from two settings. Diabetes Care 1999;22(1):157-62. [PubMed: 10333919]

4. McMahon MM, Bistrian BR. Host defenses and susceptibility to infection in patients with diabetes mellitus. Infectious disease clinics of North America 1995;9(1):1-9. [PubMed: 7769211]

5. Saeed RW, Varma S, Peng-Nemeroff T, Sherry B, Balakhaneh D, Huston J, et al. Cholinergic stimulation blocks endothelial cell activation and leukocyte recruitment during inflammation. The Journal of experimental medicine 2005;201(7):1113-23. [PubMed: 15809354]

6. Radek KA, Elias PM, Taupenot L, Mahata SK, O’Connor DT, Gallo RL. Neuroendocrine nicotinic receptor activation increases susceptibility to bacterial infections by suppressing antimicrobial peptide production. Cell host \& microbe 2010;7(4):277-89. [PubMed: 20413096]

7. Grando SA, Horton RM, Pereira EF, Diethelm-Okita BM, George PM, Albuquerque EX, et al. A nicotinic acetylcholine receptor regulating cell adhesion and motility is expressed in human keratinocytes. The Journal of investigative dermatology 1995;105(6):774-81. [PubMed: 7490471]

8. Glaser R, Kiecolt-Glaser JK, Marucha PT, MacCallum RC, Laskowski BF, Malarkey WB. Stressrelated changes in proinflammatory cytokine production in wounds. Archives of general psychiatry 1999;56(5):450-6. [PubMed: 10232300]

9. Dong MW, Li M, Chen J, Fu TT, Lin KZ, Ye GH, et al. Activation of alpha7nAChR Promotes Diabetic Wound Healing by Suppressing AGE-Induced TNF-alpha Production. Inflammation 2016;39(2):687-99. [PubMed: 26650489]

10. Nizet V, Gallo RL. Cathelicidins and innate defense against invasive bacterial infection. Scand J Infect Dis 2003;35(9):670-6. [PubMed: 14620153]

11. Ramos R, Silva JP, Rodrigues AC, Costa R, Guardao L, Schmitt F, et al. Wound healing activity of the human antimicrobial peptide LL37. Peptides 2011;32(7):1469-76. [PubMed: 21693141]

12. Aberg KM, Radek KA, Choi EH, Kim DK, Demerjian M, Hupe M, et al. Psychological stress downregulates epidermal antimicrobial peptide expression and increases severity of cutaneous infections in mice. J Clin Invest 2007;117(11):3339-49. [PubMed: 17975669]

13. Lai Y, Cogen AL, Radek KA, Park HJ, Macleod DT, Leichtle A, et al. Activation of TLR2 by a small molecule produced by Staphylococcus epidermidis increases antimicrobial defense against bacterial skin infections. The Journal of investigative dermatology 2010;130(9):2211-21.

[PubMed: 20463690] 
14. Schauber J, Dorschner RA, Coda AB, Buchau AS, Liu PT, Kiken D, et al. Injury enhances TLR2 function and antimicrobial peptide expression through a vitamin D-dependent mechanism. J Clin Invest 2007;117(3):803-11. [PubMed: 17290304]

15. Johnson CM, Tapping RI. Microbial products stimulate human Toll-like receptor 2 expression through histone modification surrounding a proximal NF-kappaB-binding site. The Journal of biological chemistry 2007;282(43):31197-205. [PubMed: 17728249]

16. Jimenez-Dalmaroni MJ, Xiao N, Corper AL, Verdino P, Ainge GD, Larsen DS, et al. Soluble CD36 ectodomain binds negatively charged diacylglycerol ligands and acts as a co-receptor for TLR2. PloS one 2009;4(10):e7411. [PubMed: 19847289]

17. van Bergenhenegouwen J, Plantinga TS, Joosten LA, Netea MG, Folkerts G, Kraneveld AD, et al. TLR2 \& Co: a critical analysis of the complex interactions between TLR2 and coreceptors. Journal of leukocyte biology 2013;94(5):885-902. [PubMed: 23990624]

18. Dasu MR, Devaraj S, Park S, Jialal I. Increased toll-like receptor (TLR) activation and TLR ligands in recently diagnosed type 2 diabetic subjects. Diabetes Care 2010;33(4):861-8. [PubMed: 20067962]

19. Dasu MR, Thangappan RK, Bourgette A, DiPietro LA, Isseroff R, Jialal I. TLR2 expression and signaling-dependent inflammation impair wound healing in diabetic mice. Laboratory investigation; a journal of technical methods and pathology 2010;90(11):1628-36. [PubMed: 20733560]

20. Wang X, Yang Z, Xue B, Shi H. Activation of the cholinergic antiinflammatory pathway ameliorates obesity-induced inflammation and insulin resistance. Endocrinology 2011;152(3):83646. [PubMed: 21239433]

21. Kishibe M, Griffin TM, Radek KA. Keratinocyte nicotinic acetylcholine receptor activation modulates early TLR2-mediated wound healing responses. Int Immunopharmacol 2015;29(1):6370. [PubMed: 26071220]

22. Schneider CA, Rasband WS, Eliceiri KW. NIH Image to ImageJ: 25 years of image analysis. Nat Methods 2012;9(7):671-5. [PubMed: 22930834]

23. Tossi A, Sandri L, Giangaspero A. Amphipathic, alpha-helical antimicrobial peptides. Biopolymers 2000;55(1):4-30. [PubMed: 10931439]

24. Radek KA, Elias PM, Taupenot L, Mahata SK, O'Connor DT, Gallo RL. Neuroendocrine nicotinic receptor activation increases susceptibility to bacterial infections by suppressing antimicrobial peptide production. Cell Host and Microbe 2010;7:277-89. [PubMed: 20413096]

25. Acosta JB, del Barco DG, Vera DC, Savigne W, Lopez-Saura P, Guillen Nieto G, et al. The proinflammatory environment in recalcitrant diabetic foot wounds. International wound journal 2008;5(4):530-9. [PubMed: 19006574]

26. Bader MS. Diabetic foot infection. American family physician 2008;78(1):71-9. [PubMed: 18649613]

27. Dasu MR, Martin SJ. Toll-like receptor expression and signaling in human diabetic wounds. World journal of diabetes 2014;5(2):219-23. [PubMed: 24748934]

28. Raby AC, Le Bouder E, Colmont C, Davies J, Richards P, Coles B, et al. Soluble TLR2 reduces inflammation without compromising bacterial clearance by disrupting TLR2 triggering. Journal of immunology 2009;183(1):506-17.

29. Chen Y, Guarnieri MT, Vasil AI, Vasil ML, Mant CT, Hodges RS. Role of peptide hydrophobicity in the mechanism of action of alpha-helical antimicrobial peptides. Antimicrob Agents Chemother 2007;51(4):1398-406. [PubMed: 17158938]

30. LeBouder E, Rey-Nores JE, Rushmere NK, Grigorov M, Lawn SD, Affolter M, et al. Soluble forms of Toll-like receptor (TLR) 2 capable of modulating TLR2 signaling are present in human plasma and breast milk. Journal of immunology 2003;171(12):6680-9.

31. Kuroishi T, Tanaka Y, Sakai A, Sugawara Y, Komine K, Sugawara S. Human parotid saliva contains soluble toll-like receptor (TLR) 2 and modulates TLR2-mediated interleukin-8 production by monocytic cells. Molecular immunology 2007;44(8):1969-76. [PubMed: 17081611]

32. Liew FY, Xu D, Brint EK, O’Neill LA. Negative regulation of toll-like receptor-mediated immune responses. Nature reviews Immunology 2005;5(6):446-58.

Wound Repair Regen. Author manuscript; available in PMC 2019 November 01. 
33. Wetzler C, Kampfer H, Stallmeyer B, Pfeilschifter J, Frank S. Large and sustained induction of chemokines during impaired wound healing in the genetically diabetic mouse: prolonged persistence of neutrophils and macrophages during the late phase of repair. The Journal of investigative dermatology 2000;115(2):245-53. [PubMed: 10951242]

34. Ahmad J, Zubair M, Malik A, Siddiqui MA, Wangnoo SK. Cathepsin-D, adiponectin, TNF-alpha, IL-6 and hsCRP plasma levels in subjects with diabetic foot and possible correlation with clinical variables: a multicentric study. Foot 2012;22(3):194-9.

35. Rivas-Santiago B, Trujillo V, Montoya A, Gonzalez-Curiel I, Castaneda-Delgado J, Cardenas A, et al. Expression of antimicrobial peptides in diabetic foot ulcer. Journal of dermatological science 2012;65(1):19-26. [PubMed: 22047630]

36. Rehani K, Scott DA, Renaud D, Hamza H, Williams LR, Wang H, et al. Cotinine-induced convergence of the cholinergic and PI3 kinase-dependent anti-inflammatory pathways in innate immune cells. Biochimica et biophysica acta 2008;1783(3):375-82. [PubMed: 18178163]

37. Liang SC, Tan XY, Luxenberg DP, Karim R, Dunussi-Joannopoulos K, Collins M, et al. Interleukin (IL)-22 and IL-17 are coexpressed by Th17 cells and cooperatively enhance expression of antimicrobial peptides. The Journal of experimental medicine 2006;203(10):2271-9. [PubMed: 16982811]

38. Kumar P, Natarajan K, Shanmugam N. High glucose driven expression of pro-inflammatory cytokine and chemokine genes in lymphocytes: molecular mechanisms of IL-17 family gene expression. Cellular signalling 2014;26(3):528-39. [PubMed: 24308966]

39. Jacobi J, Jang JJ, Sundram U, Dayoub H, Fajardo LF, Cooke JP. Nicotine accelerates angiogenesis and wound healing in genetically diabetic mice. The American journal of pathology 2002;161(1): 97-104. [PubMed: 12107094] 
A

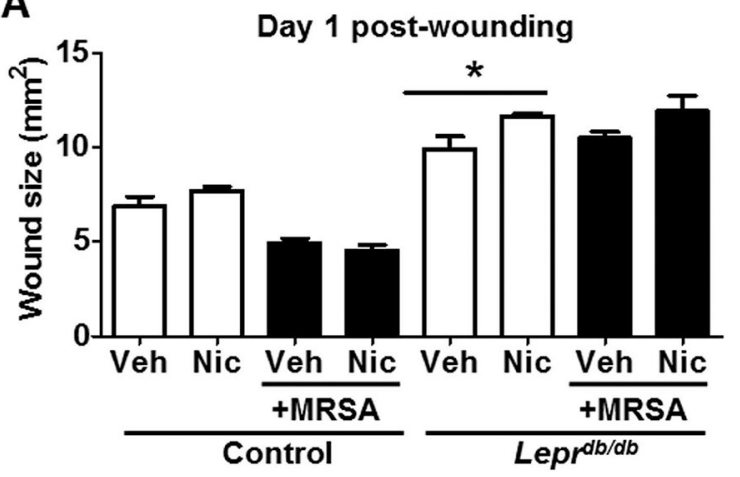

C

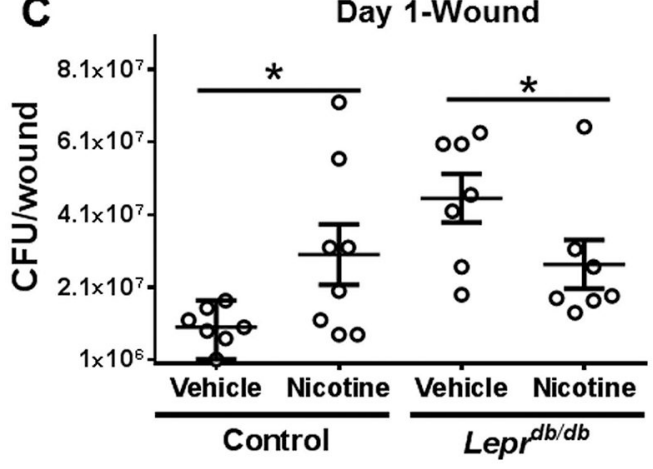

B

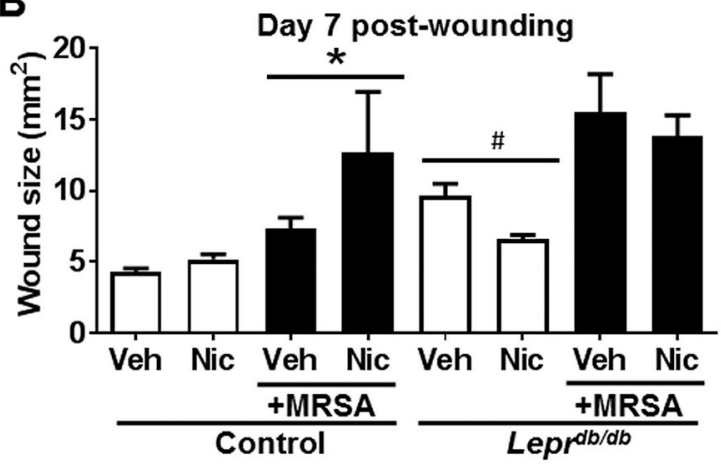

D

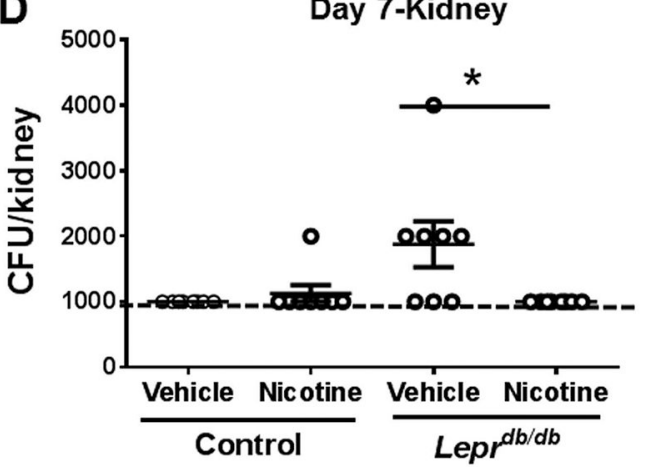

Figure 1. Epidermal nAChR activation influences wound closure and bacterial survival in diabetic mouse wounds.

Vehicle (Veh) or nicotine (Nic; $10^{-9} \mathrm{M}$ ) was applied twice daily onto dorsal skin for 3 days prior to excisional wounding and topical vehicle or infection with $10^{6} \mathrm{CFU} /$ wound of MRSA. Wound sizes of uninfected or infected control (Lepr $d b /+)$ or diabetic $\left(L e p r^{d b / d b}\right)$ mice were determined at (A) day 1 and (B) day 7 post-wounding. Statistical analyses were performed using a Mann Whitney test $\left({ }^{*}, p<0.05,{ }^{\#}, p<0.01\right)$. (C) Bacterial burden in skin wounds at day 1 post-wounding and (D) MRSA dissemination into kidney at day 7 postwounding in control $\left(\operatorname{Lepr}^{d b /+}\right)$ and diabetic Lepr ${ }^{d b / d b}$ mice ( $n=7-8$ per group, repeated twice). Representative data from one of the two independent experiments are shown. Dotted line in (D) demarcates detection limit for bacteria in this assay $\left(10^{3} \mathrm{CFU} /\right.$ kidney). Data are presented as the median \pm range. Each dot indicates a single mouse. Statistical analysis was performed using unpaired one-tailed Mann-Whitney test $(* p<0.05$ vs. vehicle for respective control or diabetic mouse). 

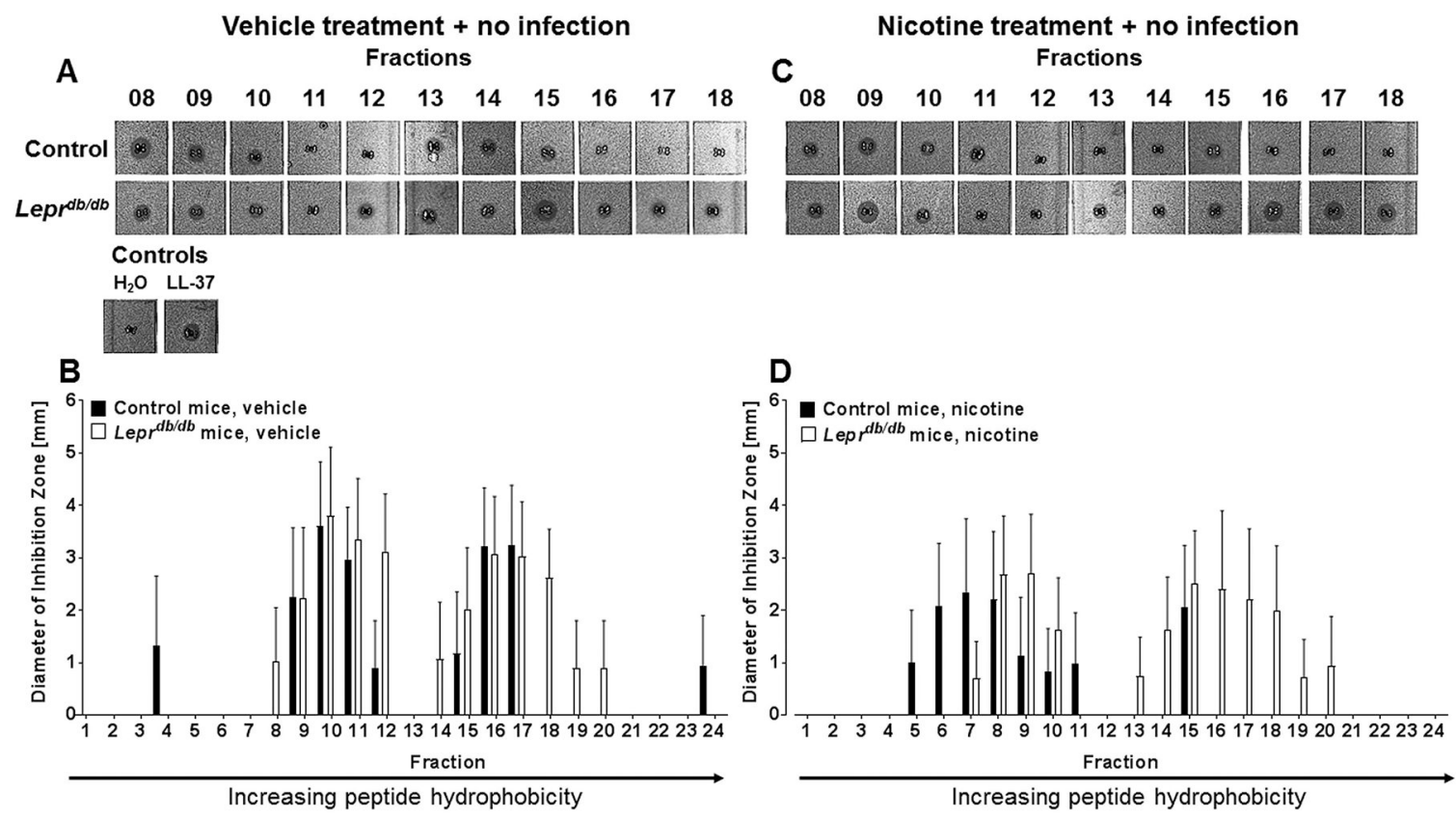

Figure 2. Topical nAChR activation does not alter AMP activity or hydrophobicity in uninfected mouse wounds from control or diabetic mice.

Wounds from vehicle treated (A-B) or nicotine treated (C-D) control Lepr ${ }^{d b /+}$ and spontaneous diabetic $L e p r^{d b / d b}$ mice were excised at day 1 post-wounding and subjected to HPLC fractionation and radial diffusion assay. Bacterial growth inhibition diameters were measured using ImageJ software. Data were statistically analyzed by Mann-Whitney test. $\mathrm{N}=4-5$ samples/group. 


\section{A} Vehicle treatment + MRSA infection

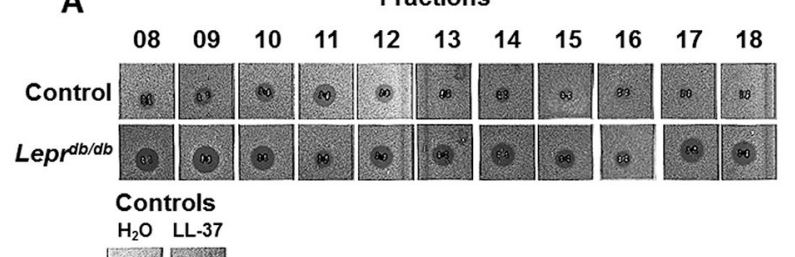

C

Nicotine treatment + MRSA infection

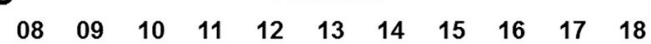

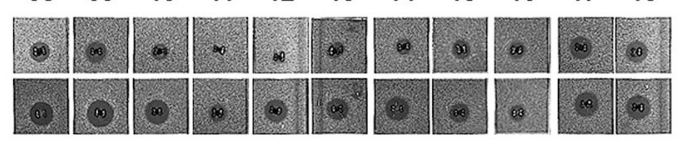

B
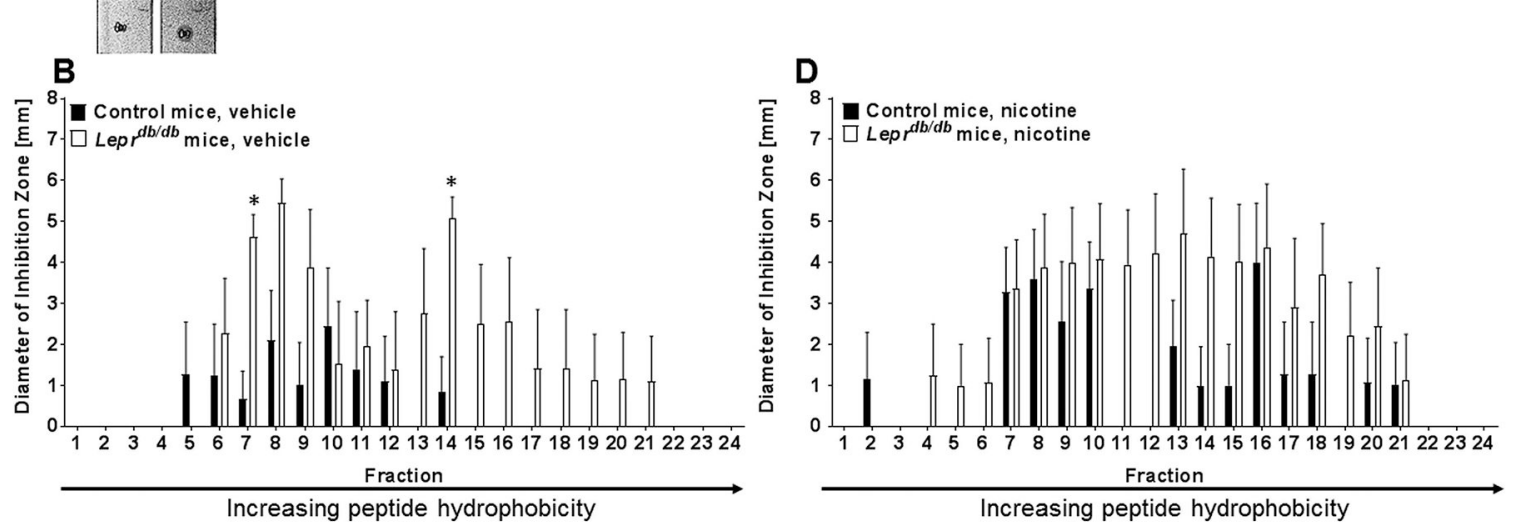

Figure 3. Topical nAChR activation normalizes AMP activity and hydrophobicity in MRSAinfected mouse wounds in diabetic mice.

Wounds from vehicle treated (A-B) or nicotine treated (C-D) control $\mathrm{Lepr}^{\mathrm{db} / \mathrm{t}}$ and spontaneous diabetic $L e p r^{d b / d b}$ mice were infected with MRSA bacteria and excised at day 1 post-wounding/infection and subjected to HPLC fractionation and radial diffusion assay. Bacterial growth inhibition diameters were measured using ImageJ software. Data were statistically analyzed by ANOVA and Sidak's post-test (*, $p<0.05$ vs. control mouse fraction). $\mathrm{N}=4-5$ samples/group. 
A

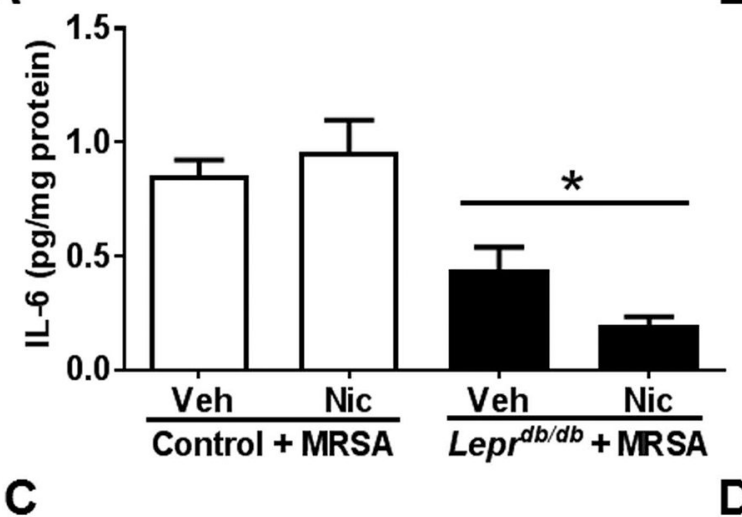

B

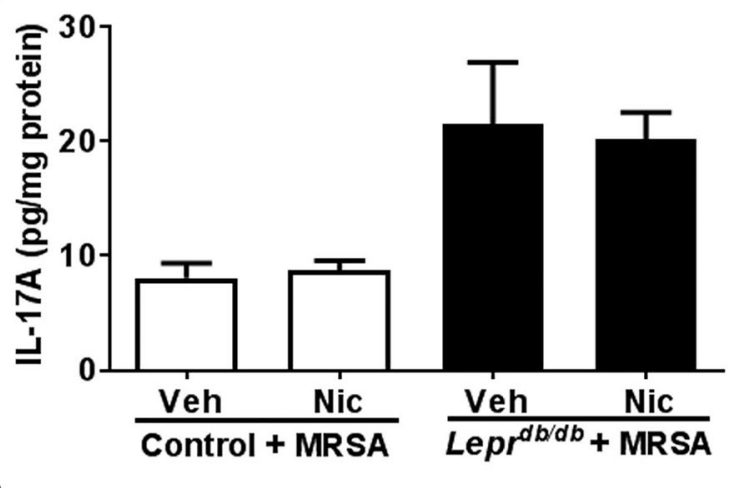

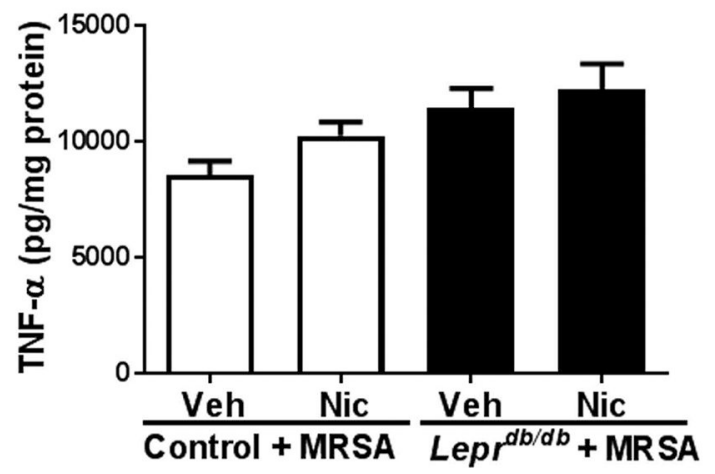

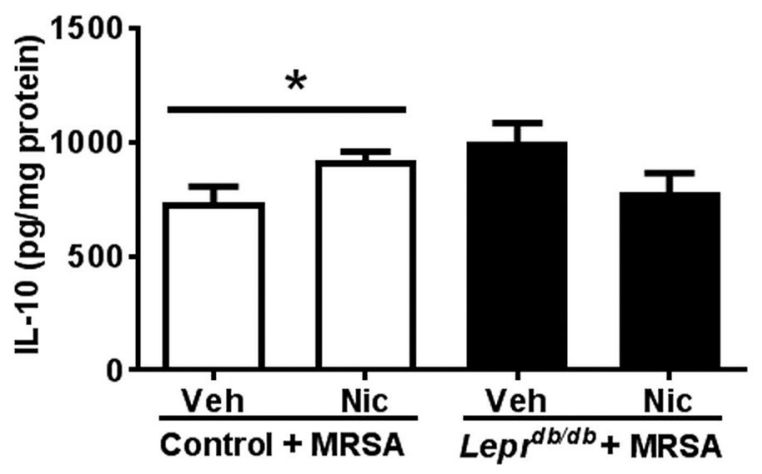

Figure 4. Topical nAChR activation decreases IL-6 production in MRSA infected diabetic mouse wounds, and slightly increases IL-10 in MRSA infected control mouse wounds.

The concentrations of (A) IL-6, (B) IL-17A, (C) TNF-a and (D) IL-10 at day 1 post-

wounding were measured by ELISA in homogenates of MRSA infected skin wounds with or without nicotine treatment of control mice $\left(L e p r^{d b /+}\right.$ ) or diabetic mice $\left(L e p r^{d b / d b}\right.$ ) ( $n=8$ for each group). Data are presented as the mean \pm sem. Statistical analyses were performed using Mann-Whitney one-tailed t-test ( $\left.{ }^{*}, p<0.05\right)$. Veh, vehicle; Nic, nicotine $\left(10^{-9} \mathrm{M}\right)$. 

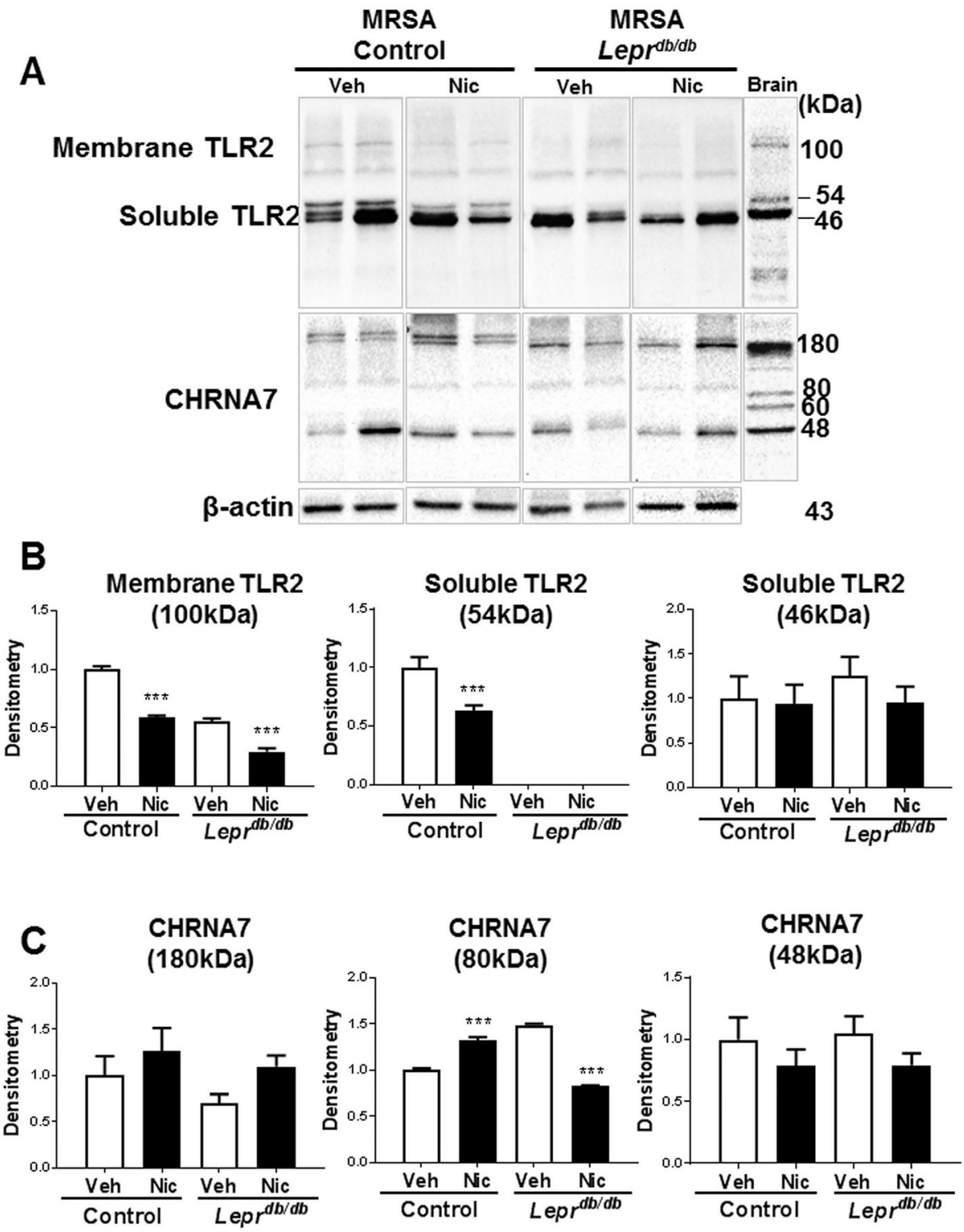

Figure 5. Membrane TLR2 and CHRNA7 protein levels are reduced by nicotine treatment in MRSA infected diabetic mouse wounds.

(A) Expression of TLR2, CHRNA7, and $\beta$-actin in skin of control $\left(\mathrm{Lepr}^{\mathrm{db} /+}\right)$ or diabetic $\left(L e p r^{d b / d b}\right.$ ) mice at day 1 post wounding were detected by Western Blot ( $n=8$ per group, repeated twice). C57B1/6 mouse brain lysate was used as positive control. Semi-quantitative expression of membrane and soluble TLR2 (B) and CHRNA7 (C) was determined by densitometry after normalization to the internal control band ( $\beta$-actin). Data are presented as the mean \pm sem. Statistical analysis was performed using one way ANOVA and Bonferroni's post-test adjusted $P$ value (***, $p<0.001$ ). Veh, vehicle treatment; Nic, nicotine treatment $\left(10^{-9} \mathrm{M}\right)$. 